\title{
Exact closed-form solution to jet flow of a polymer arising in the electrospun nanofiber elaboration process
}

\author{
Robert A. Van Gorder* \\ Mathematical Institute, University of Oxford \\ Andrew Wiles Building, Radcliffe Observatory Quarter, Woodstock Road, Oxford, OX2 6GG, United Kingdom \\ Email: Robert.VanGorder@maths.ox.ac.uk
}

August 11, 2015

\begin{abstract}
In the present paper, we obtain the exact solution to the jet flow of a polymer arising in the electrospun nanofiber elaboration process. This allows us to improve on the approximate solutions of Colantoni \& Boubaker (2014) and He et al. (2014). Our corrected and far simpler solution is more useful for extracting the salient physics inherent in the electrospun nanofiber elaboration process. We then consider the more robust formulation of the problem, in which the velocity of the jet and the radius of the jet depend strongly on one another, obtaining the solution in this case as well. All of the results obtained are rather simple, and give us closedform exact solutions (rather than complicated approximations), and hence these results both maintain accuracy and have the possibility of being useful for future studies into the physics of such mechanical processes. The results also agree nicely with experimental and numerical results present in the literature.
\end{abstract}

Keywords: Exact solution; Electrospinning; Vibration-electrospinning; Jet flow

\section{Introduction}

Electrospinning is a process proposed by Formhals (Formhals, 1934) for elaborating nanofibers with diameters about $20 \mathrm{~nm}$ by forcing a fluidified polymer through a spinneret by an electric field. Under the influence of the electrostatic field, a pendant droplet of the polymer solution at the capillary tip, at the outer edge of a controlled syringe, is deformed into a conical shape, namely a Taylor cone (Taylor, 1954). If the voltage surpasses a threshold value, electro-static forces overcome the surface tension, and a charged fine jet is ejected (Doshi \& Reneker, 1995; Fong \& Reneker, 2001; Gibsonet al., 1999). This jet moves toward a collector grid. The main controlling parameters of the process are hydrostatic pressure in the capillary tube and external electric field, material viscosity, conductivity, dielectric permeability, surface tension, and temperature gradient. This process is of great interest to the polymer industry. In this paper, we shall focus on the dynamics of the jet. A schematic of the jet motion, from the pump apparatus to the collector grid, is shown in Figure 1.

The mechanical equations which govern the electrospinning process (Spivak \& Dzenis, 1998; Spivak, Dzenis, \& Reneker, 2000) are mass balance, linear momentum balance and electric charge balance equations respectively. Starting with these, and assuming a weak dependence of the jet 


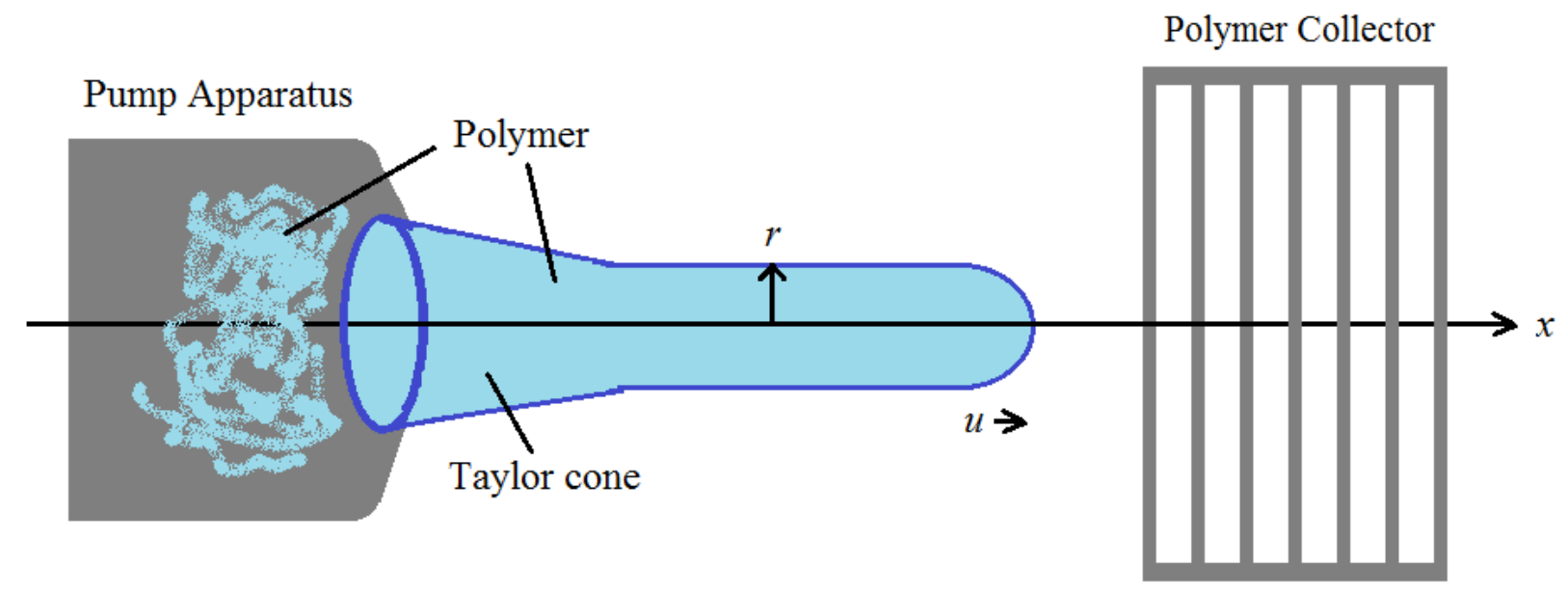

Figure 1: Schematic of the jet motion and problem geometry. After being ejected from the pump apparatus under the influence of the electrostatic field, the carbohydrate polymer forms a Taylor cone which then shrinks in radius and gives us a jet. This jet then propagates toward the collector. The jet is oriented along the $x$ axis and propagates with velocity $u$. The radius, $r$, of the jet can be assumed constant in $x$ for some applications, although for other applications (as will be discussed in Section 4) the jet radius can vary in $x$.

radius, $r$, on the spatial position $x$ (Spivak et al., 2000), Colantoni \& Boubaker (2014) derive a type of Bratu equation governing this process. They then solved this equation using EVIM and BPES, two analytical methods for solving differential equations. However, He et al. (2014) pointed out that the formulation of Colantoni \& Boubaker (2014) is incorrect.

Using far simpler techniques than Colantoni \& Boubaker (2014), we obtain an exact solution to their model in Section 2. Now, given that He et al. (2014) point out an error in the formulation of Colantoni \& Boubaker (2014), we also obtain exact solutions for the correct version of the problem, in Section 3. For cases considered by both Colantoni \& Boubaker (2014) and He et al. (2014), a weak dependence of $r$ on $x$ was assumed, meaning that $r$ was taken to be a constant. We show that in general this is not true, and we then solve the more useful model resulting from taking $r$ as a function of $x$. In this case, we obtain an equation for $r(x)$, and once solved this gives the solution for the velocity, $u(x)$. In all cases considered, one may obtain an exact solution, hence there is no real reason to map the problem into something like a Bratu equation and then attempt to find an approximate solution. We feel as though these exact solutions will be of far greater use to researchers interested in the actual physical processes at work in this problem.

The purpose of the paper was to improve on the approximations of Colantoni \& Boubaker (2014) and He et al. (2014). All of the results obtained are rather simple, and give us closed-form exact solutions (rather than complicated approximations), and hence these results both maintain accuracy and have the possibility of being useful for future studies into the physics of such mechanical processes. Furthermore, we ensure that the model solved is the correct one (as He et al. (2014) pointed out, Colantoni \& Boubaker (2014) did not really solve the correct problem). Hence, the present results may be of use to those interested in studying the jet flow problem in this context. 


\section{Formulation of Colantoni \& Boubaker and the corrected exact solution}

We first follow the derivations of Colantoni \& Boubaker (2014). The electro-spinning process is governed by the mass balance equation

$$
\nabla \cdot \mathbf{u}=0
$$

the linear momentum balance equation

$$
\rho(\mathbf{u} \cdot \nabla) \mathbf{u}=\nabla F^{m}+\nabla F^{e},
$$

and the electric charge balance equation

$$
\nabla \cdot \mathbf{j}=0 .
$$

Here $\mathbf{u}$ is the axial velocity, $\mathbf{j}$ is the electric current density, $\rho$ is the material density, $F^{m}$ is the viscous force term, and $F^{e}$ is the electric force term. If we consider a steady jet and ignore thermal effects, the momentum equation reduces to

$$
u \frac{\partial u}{\partial x}=\frac{2 \sigma E}{\rho r} .
$$

Where $u$ is the modulus of the axial velocity, $r$ is the radius of the jet at axial coordinate $x, \sigma$ is the surface charge density, and $E$ is electric field in the axial direction. Colantoni \& Boubaker (2014) consider the charge balance equation $2 r \sigma E+r^{2} k E=I$, where $I$ is the electrical current intensity and $k$ is a constant which depends only on the temperature in the case of an incompressible polymer. With this, (5) is put into the form

$$
u \frac{\partial u}{\partial x}=\frac{E\left(I-r^{2} k E\right)}{\rho r^{2}}=A,
$$

where we have introduced the constant $A=\frac{E\left(I-r^{2} k E\right)}{\rho r^{2}}$ for simplicity. Integrating (5) with respect to $x$, we immediately find

$$
u(x)^{2}=A x+B^{2},
$$

where the constant $B^{2}$ corresponds to the condition $u=B$ at $x=0$. The exact solution is immediately found to be

$$
u(x)=\sqrt{A x+B^{2}} .
$$

This solution exists on the domain $-1 \leq x \leq 1$ provided that $B^{2} \geq|A|$. If $|A|>B^{2}$, it is clear that no solution will exist.

Note that this solution was found in exact form immediately after integration of (5), and hence pages of lengthy and unneeded calculations were avoided.

\subsection{The Bratu equation approach}

In Colantoni and Boubaker (2014), (5) was converted into a Bratu differential equation and then solved via two analytical approaches. The authors transformed the equation by introducing a new dependent variable

$$
y(x)=-6 \ln (u(x))
$$


However, this transformation is not consistent with equation (9) of Colantoni and Boubaker (2014). Indeed, if one uses this transformation, it is clear that

$$
u(x)=\exp \left(-\frac{1}{6} y(x)\right) \quad \text { and } \quad u^{\prime}(x)=-\frac{1}{6} \exp \left(-\frac{1}{6} y(x)\right) y^{\prime}(x) .
$$

This gives (from (5)) the equation $y^{\prime}=-6 A \exp (y / 3)$, which is not what is shown in equation (9) of Colantoni and Boubaker (2014). Hence, the remaining results they obtain are inconsistent with the original electrospinning problem.

Note also that Colantoni and Boubaker (2014) pick the conditions $y(0)=0$ and $y^{\prime}(0)=0$. Yet, from what we show above, if $y(0)=0$, then $y^{\prime}(0)=-6 A=0$ so $A=0$. Then, $y^{\prime}=0$ for all $x$, hence $y(x)$ is a constant. Yet, $y(0)=0$, so $y(x)=0$ for all $x$. In light of the transformation (8), this would mean that $u(x)=1$ for all $x$. This is a trivial constant solution. In other words, the conditions $y(0)=0$ and $y^{\prime}(0)=0$ taken in Colantoni and Boubaker (2014) are unreasonable for the present problem, and can only result in a constant solution. Conversely, they do not show on constant solution, meaning they have ended up solving some different problem than the electrospinning problem.

\subsection{Recovery of the correct solution to (5)}

If we instead use the transform

$$
y(x)=-4 \ln (u(x)),
$$

we obtain

$$
u(x)=\exp \left(-\frac{1}{4} y(x)\right) \quad \text { and } \quad u^{\prime}(x)=-\frac{1}{4} \exp \left(-\frac{1}{4} y(x)\right) y^{\prime}(x) .
$$

This results in a differential equation of the form $y^{\prime}=-4 A \exp (y / 2)$. Differentiating and putting the equation into the proper form, we obtain

$$
y^{\prime \prime}-8 A^{2} \exp (y)=0,
$$

which is indeed a Bratu equation. In order to obtain the proper initial conditions, let us note that

$$
y(0)=-4 \ln (u(0)) \quad \text { and } \quad y^{\prime}(0)=-4 A \exp (-2 \ln (u(0)))=-\frac{4 A}{u(0)^{2}} .
$$

These values each depend on $u(0)$ and hence are related. In particular, they cannot simply be set to zero.

Now that we have the proper and physically meaningful Bratu equation (12) subject to relevant initial conditions (13), we can solve the problem. Multiplying (12) by $2 y^{\prime}$ and integrating in the variable $x$ we obtain

$$
y^{\prime 2}-16 A^{2} \exp (y)=y^{\prime}(0)^{2}-16 A^{2} \exp (y(0))=\frac{16 A^{2}}{u(0)^{4}}-\frac{16 A^{2}}{u(0)^{4}}=0 .
$$

Therefore, $y^{\prime 2}-16 A^{2} \exp (y)=0$. This equation is separable, viz.,

$$
\frac{y^{\prime 2}}{16 A^{2} \exp (y)}=1 \Rightarrow \pm \frac{y^{\prime}}{4 A \exp (y / 2)}=1 \text {. }
$$


For physical reasons, we shall take the negative root. Performing the separation of variables,

$$
\int_{y(0)}^{y(x)} \frac{d Y}{4 A \exp (Y / 2)}=x \Rightarrow \exp (-y(x) / 2)-\exp (-y(0) / 2)=2 A x .
$$

This gives

$$
\exp (-y(x) / 2)=2 A x+u(0)^{2} .
$$

Yet, since $u(x)=\exp (-y(x) / 4)$, we have $u(x)^{2}=\exp (-y(x) / 2)$, which gives $u(x)^{2}=2 A x+u(0)^{2}$. Taking the positive root, we obtain

$$
u(x)=\sqrt{2 A x+u(0)^{2}},
$$

which is exactly the solution we obtained via the more direct route in (7). Thus, while the Bratu formulation is a bit more work, it should (and, does) result in the same solution as found via the direct approach.

\section{Formulation of $\mathrm{He}$ et al. and exact solution}

As pointed out in He et al. (2014), the formulation of Colantoni \& Boubaker (2014) neglects a term depending on $u$, in equation (5). The proper formulation was previously studied in Wan et al. (2004). In particular, the charge balance equation should have been $2 r \sigma u+r^{2} k E=I$. In this case, one obtains

$$
u \frac{\partial u}{\partial x}=\frac{E\left(I-r^{2} k E\right)}{\rho r^{2} u}
$$

or

$$
u^{2} \frac{\partial u}{\partial x}=\frac{E\left(I-r^{2} k E\right)}{\rho r^{2}} .
$$

Note that in this formulation, one assumes that the dependence of $r$ on $x$ is negligible. This was true of the case considered by Colantoni \& Boubaker (2014), as well. Therefore, treating $x$ and $r$ as independent of one another, and integrating (20) with respect to $x$, we obtain

$$
u(x)^{3}-u(0)^{3}=\frac{3 E\left(I-r^{2} k E\right)}{\rho r^{2}} x,
$$

hence

$$
u(x)=\sqrt[3]{\frac{3 E\left(I-r^{2} k E\right)}{\rho r^{2}} x+u(0)^{3}} .
$$

This is the exact solution for (20). Again, note that no complicated method is required to obtain this solution. Due to the proper inclusion of $u$ in the charge balance equation, which is correct, we have obtained a cubed-root solution. In contrast, by incorrectly dropping the functional dependence on $u$ in the charge balance equation, the equation resulting from the formulation of Colantoni \& Boubaker (2014) has a solution which scales as a square root. 


\section{The situation where the jet radius varies in space}

While both Colantoni \& Boubaker (2014) and He et al. (2014) consider the situation where the relation between $r$ and $x$ is negligible, note that this is not always true (Spivak et al., 2000). Therefore, we should attempt to make sure that any results are consistent with the more general case where $r$ does indeed depend on $x$. Assuming $r=r(x)$, the charge balance equation is

$$
2 \sigma r(x) u(x)+r^{2}(x) k E=I,
$$

hence

$$
u(x)=\frac{I-E k r(x)^{2}}{2 \sigma r(x)}=\frac{I}{2 \sigma r(x)}-\frac{E k}{2 \sigma} r(x) .
$$

Therefore, the momentum equation can be put into the form

$$
\left(r(x)^{2}-\frac{I}{k E}\right)\left(r(x)^{2}+\frac{2 I}{k E}\right) \frac{r^{\prime}(x)}{r(x)^{2}}=\frac{8 \sigma^{3}}{\rho k^{2} E} .
$$

Defining the constants

$$
\mu=\frac{I}{k E} \quad \text { and } \quad \nu=\frac{8 \sigma^{3}}{\rho k^{2} E}
$$

we have

$$
\left(r^{2}-\mu\right)\left(r^{2}+2 \mu\right) \frac{r^{\prime}}{r^{2}}=\nu .
$$

This equation has a first integral,

$$
\frac{1}{3} r^{3}+\mu r+\frac{2 \mu^{2}}{r}=\nu\left(x-x_{0}\right),
$$

where $x_{0}$ is an integration constant. Let us define a new function $R$ and variable $X$ by

$$
r(x)=\sqrt{3 \mu} R(X) \quad \text { where } \quad X=\frac{\nu}{\sqrt{3 \mu^{3}}}\left(x-x_{0}\right) .
$$

Then, (28) can be written in the form

$$
R^{4}+R^{2}-X R+\frac{2}{3}=0
$$

For $X>0$, there are two solutions: one upper solution branch, and one lower solution branch. This is shown in Figure 1. For physical reasons, one should select the increasing solution branch (the upper solution branch).

The exact solution to (30) can be obtained on a computer algebra system, such as Maple, since the quartic equation is exactly solvable. We obtain the larger root in Figure 1 by the formula

$$
R(X)=\frac{1}{2 \sqrt{6}} \sqrt[3]{q(X)+\frac{36}{q(X)}-4}+\frac{1}{2 \sqrt{6}} \sqrt[3]{\frac{12 \sqrt{6} X}{q(X)+\frac{36}{q(X)}-4}-q(X)-\frac{36}{q(X)}-8},
$$

where

$$
q(X)=4 \sqrt{\left(27 X^{2}-100\right)\left(27 X^{2}+8\right)}+108 X^{2}-184 .
$$




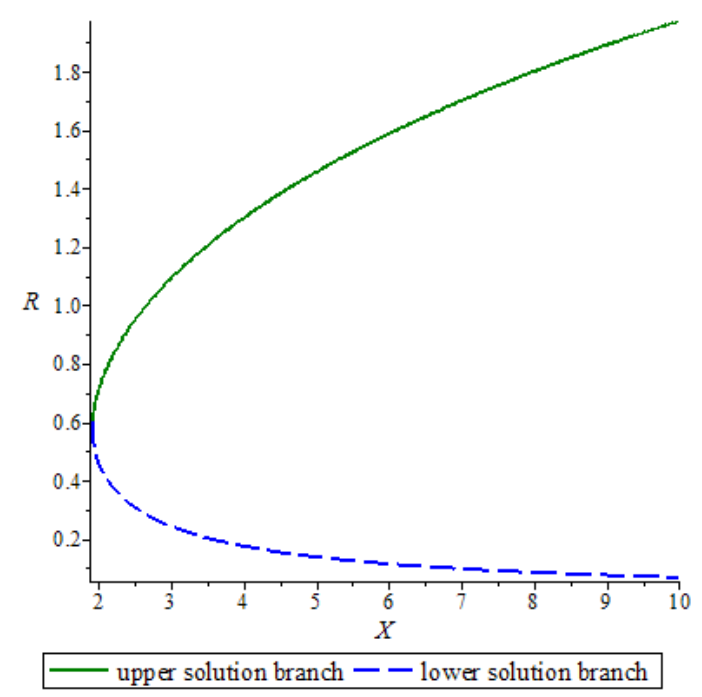

Figure 2: Plot of the upper and lower solution branches obtained from solving the quartic equation (30) for $R$ as a function of $X$. Two other branches have non-zero complex part and hence are not physically relevant.

This solution is real-valued for $X>\frac{10}{3 \sqrt{3}}$. So, one should pick the arbitrary constant $x_{0}$ such that

$$
\frac{\nu}{\sqrt{3 \mu^{3}}}\left(x-x_{0}\right)>\frac{10}{3 \sqrt{3}} .
$$

Note that for some applications, it may be necessary to select the lower solution branch shown in Figure 1. One obtains a slightly different formula for $R(X)$. This selection will depend on if the radius of the jet widens or thins with increasing $x$. This is particularly true when the jet radius must decrease asymptotically, in which case we must take the lower branch.

While the exact solution (31) is complicated, it is possible to study the structure of a solution for large $X$, in a far less complicated way. If we assume the existence of a dominant term, say

$$
R(X) \sim X^{p}+\text { lower order terms }
$$

then by placing this expression into (30) we find that the dominant term should scale like $X^{3 p-1} \sim 1$, hence $p=\frac{1}{3}$. As such, $R(X)$ scales like

$$
R(X) \sim \sqrt[3]{X}+\text { lower order terms },
$$

for large $X$, and therefore $r(x)$ scales like

$$
r(x) \sim \sqrt[3]{x}+\text { lower order terms },
$$

for large $x$. This scaling is in complete agreement with what we see in Figure 1. 
Finally, from (24) and (29) we can obtain the dependence of $u(x)$ on the function $R(X)$, which gives

$$
u(x)=\frac{\sqrt{k E I}}{2 \sigma}\left\{\frac{1}{\sqrt{3} R\left(\sqrt{\frac{E}{3 k I^{3}}} \frac{8 \sigma^{3}}{\rho}\left(x-x_{0}\right)\right)}-\sqrt{3} R\left(\sqrt{\frac{E}{3 k I^{3}}} \frac{8 \sigma^{3}}{\rho}\left(x-x_{0}\right)\right),\right.
$$

where again $R$ is the relevant real branch of the quartic equation (30) shown in Figure 1. Then, $u(x)$ has the scaling

$$
u(x) \sim \sqrt[3]{x}+\text { lower order terms },
$$

for large $x$. This is completely consistent with what we have recovered from the exact solution (22). Therefore, the assumption that $r$ is independent of $x$ gives an exact solution that is asymptotically consistent with the more general case in which $r$ does indeed vary as a function of $x$. If the lower branch in Figure 1 is taken, then the velocity is positive and increases in space, while the radius of the jet decreases.

\section{Conclusions}

We have been able to demonstrate that the solutions to the jet flow resulting from the electrospinning process for carbohydrate polymers described above can actually be obtained in the form of closed-form exact solutions in the case where the radius of the jet is constant in space. Such solutions are rather useful for those interested in the physics of the electro-spinning problem, as they allow for one to easily understand qualitative properties of the solutions. In contrast to other attempts in the literature, the solutions are exact rather than approximate, and the solution expressions are actually rather simple. He et al. (2014) pointed out that, in addition to the mathematical problems of Colantoni \& Boubaker (2014), the formulation of that paper was also flawed. We have used the correct formulation to obtain the exact solution of the problem proposed in He et al. (2014), which is really the same problem proposed in Wan et al. (2004). Again, we find that an exact solution can be given in rather concise form, and that no complicated approximation method is required.

We also consider the case in which the jet radius strongly depends on the spatial location, $x$. This is in contrast to the works of Colantoni \& Boubaker (2014) and He et al. (2014), where the jet radius was assumed constant. We obtain the exact solution for the radius in terms of $x$, and then we use this to recover the exact solution for the modulus of the axial velocity, $u(x)$.

In addition to the exact solutions, which may be of some relevance to those interested in the mechanics of the electro-spinning process, the results demonstrate that one should be careful when applying various approximation methods present in the literature. Indeed, it is rather easy to obtain unreasonable solutions if one does not properly check that such solutions agree with the physics of the problem at hand. Hence, while certain approximation methods are certainly useful when applied correctly, one must be mindful to verify that obtained solutions do agree and correspond with the physics of the problem.

With this, we have improved on the approximations of Colantoni \& Boubaker (2014) and He et al. (2014), obtaining exact solutions to the jet flow resulting from the electro-spinning process for carbohydrate polymers. Additionally, we have obtained results for the case in which the jet radius varies in space. These results may be useful for those interested in the physics of the electrospinning process for carbohydrate polymers, as such solutions can motivate further work, in addition 
to serving as a baseline of comparison with any numerical or experimental data. The solutions, particularly those valid for jets of spatially varying radius, may motivate the consideration of more detailed yet qualitatively more accurate models.

\subsection{Acknowledgments}

The authors wishes to thank the reviewers for helpful comments which have improved the quality of the paper.

\section{References}

[1] Colantoni, A., \& Boubaker, K. (2014) electro-spun organic nanofibers elaboration process investigations using comparative analytical solutions. Carbohydrate Polymers, 101, 307-312.

[2] Doshi, J., \& Reneker, D. H. (1995). Electrospinning process and application of electrospun fibers. Journal of Electrostatics, 35, 151-159.

[3] Fong, H., \& Reneker, D. H. (2001). Electrospinning and the formation of nanofibers.In D. R. Salem (Ed.), Structure Formation in Polymeric Fibers. Cincinnati: Hanser.

[4] Formhals, A. US Patent, 1975504, (1934).

[5] Gibson, P. W., Schreuder-Gibson, H. L., \& Rivin, D. (1999). Electrospun fiber mats: transport properties. AIChE Journal, 45, 190-196.

[6] He, J. H., Kong, H. Y., Chen, R. X., Hu, M. S., \& Chen, Q. L. (2014). Variational iteration method for Bratu-like equation arising in electrospinning. Carbohydrate Polymers, 105, 229230 .

[7] Spivak, A. F., \& Dzenis, Y. A. (1998). Asymptotic decay of radius of a weakly conductive viscous jet in an external electric field. Applied Physics Letters, 73, 3067-3069.

[8] Spivak, A. F., Dzenis, Y. A., \& Reneker, D. H. (2000). A model of steady state jet in the electrospinning process. Mechanics Research Communications, 27, 37-42.

[9] Taylor, G. (1954). Conditions under which dispersion of a solute in a stream of solvent can be used to measure molecular diffusion. Proceedings of the Royal Society of London A, 225(1163), 473-477.

[10] Wan, Y. Q., Guo, Q., \& Pan, N. (2004). Thermo-electro-hydrodynamic model for electrospinning process. International Journal of Nonlinear Sciences and Numerical Simulation, 5, $5-8$. 